\title{
Stimulus selection and memory strategies in concept problems*
}

\author{
L. E. BOURNE, JR. \\ University of Colorado, Boulder, Colorado 80302
}

\begin{abstract}
Ss were allowed to use zero, one, two, four, or eight markers to signify the category (positive or negative) of previously selected stimuli in an attribute identification problem. The more markers available and used, the fewer trials were required for problem solution. Ss were indifferent to marker valence (positive or negative) in disjunctive problems but showed a definite preference for (the more informative) negative markers in conditionals. Performance of Ss was correlated with their tendency to move markers from lesser to more informative instances. An interpretation of results based on individual differences in information processing activities is suggested.
\end{abstract}

Providing $S$ with a running record of category assignments for some number of recently presented stimulus instance has a massive facilitative effect on overall performance in conjunctive attribute identification tasks (e.g., Bourne, Goldstein, \& Link, 1964; Pishkin \& Wolfgang, 1965). Facilitation increases with the provision of up to 10 stimulus-category pairings from previous trials, although there is relatively little savings in performance beyond four.

Instead of providing $S$ with a record of recently selected instances, suppose we make it optional. We give $\mathrm{S}$ a certain number of category markers (positive and/or negative) to be applied to, if and as he wishes, selected instances on a display board. If $S$ uses the available markers, then we would certainly expect him to achieve problem solution in fewer trials. Moreover, the degree of facilitation should depend on the number of markers he uses. More importantly, however, this procedure permits an examination of S's strategy for recording information. Does $\mathbf{S}$ mark the first few stimuli selected, using them as a fixed focus for a later stimulus selection and hypothesis tests, or does he tend to move his markers from stimulus to stimulus, perhaps on the basis of judgments about the informational content of these stimuli? The classical conservative focusing strategy (Bruner, Goodnow, \& Austin, 1956; Levine, 1966) leads to the expectation that Ss who make fewer moves, focusing on an initial (few) instance(s), will reach solution in fewer trials. Instances encountered early in a sequence, however, are not necessarily the most informative. The $S$ who properly evaluates the informational content of successive instances and moves his markers consistently from stimuli with lesser to those with greater content, thus giving the appearance of a shifting focus, is likely to achieve solution more rapidly.

Another well established fact is that positive and negative instances are differentially informative across

*This research was undertaken within the Institute for the Study of Intellectual Behavior and is publication No. 38 of the Institute. The work was supported by Research Grant MH 14314 and by a Research Scientist Award, 1-K5-MH 37497, both from the National Institute of Mental Health, and by Research Grant GB 340-77X from the National Science Foundation. The author wishes to thank Katy O'Banion and Barbara Scherer for their help in collecting and analyzing the data. various conceptual rules in attribute identification problems (Bourne \& Guy, 1968). In this case, the more negative instances presented, the better is S's overall performance. The relative advantage of positive instances in disjunctive and biconditional problems is unclear, the two tending to be about equally useful (Bourne, Ekstrand, \& Montgomery, 1969).

In view of these rule and stimulus type within rule differences, it seemed prudent to compare types of memory markers (for positive or for negative instances) within at least two different rules. In this study, we elected to include both disjunctive and conditional attribute identification problems. Furthermore, under different conditions, we make available to $\mathrm{S}$ markers for positive instances only, negative instances only, or markers for both positive and negative instances. The expectation is that in disjunctive problems, where positives and negatives are about equally informative, no difference will obtain between types of markers available. When given access to both types, $S$ will choose equally between them. In the case of conditionals, where negatives are more informative than positives, however, we expect that the availability of negative markers will be more facilitative than markers for positive instances and that, given the option, $S$ will elect to use a higher proportion of negative than positive markers. We expect individual differences among $S$ s and, in this regard, Ss who elect to mark more negative instances in a conditional attribute identification problem should predictably do better than those who elect a relatively higher proportion of positive instances. Ss who exhibit a systematic shifting focus strategy should mark instances predominantly of the negative category in conditional problems. The closer $\mathrm{S}$ approximates this ideal, the fewer instances he will require for solution.

The purpose of the present study, then, is to examine S's use of memory markers for the category of previously selected instances in attribute identification problems. We expect S's strategy to relate to his overall performance in ways that are at least partly predictable. For example, we expect $S$ to make more use of negative markers in conditional problems and we expect his performance to improve with the number of markers available to him. It is not clear, however, whether 
Table 1

Mean Number of Stimulus Selections to Solution

\begin{tabular}{|c|c|c|c|c|c|}
\hline \multirow[b]{2}{*}{ Valence } & \multicolumn{5}{|c|}{ Number of Markers } \\
\hline & 0 & 1 & 2 & 4 & 8 \\
\hline \multicolumn{6}{|c|}{ Disjunctive Rule } \\
\hline+ & 22.1 & 16.1 & 15.5 & 11.9 & 9.8 \\
\hline- & 20.9 & 14.2 & 12.5 & 11.0 & 10.5 \\
\hline Both & 22.3 & 12.1 & 7.3 & 7.4 & 7.6 \\
\hline Mean & 21.8 & 14.1 & 11.4 & 9.8 & 9.0 \\
\hline \multicolumn{6}{|c|}{ Conditional Rule } \\
\hline+ & 37.1 & 32.0 & 22.1 & 12.2 & 12.0 \\
\hline- & 34.7 & 11.0 & 9.5 & 8.0 & 8.6 \\
\hline Both & 35.9 & 16.5 & 12.7 & 12.1 & 10.1 \\
\hline Mean & 35.9 & 19.8 & 14.8 & 10.8 & 10.2 \\
\hline
\end{tabular}

marking the earliest or the more recently selected stimuli will be of greater advantage. We plan to explore the effects of the rule governing problem solution and the number and the type of markers available on S's strategy. We are searching for relationships between S's strategy for using memory markers, his strategy in selecting stimuli to test, his periodic hypotheses about the solution, and his overall performance on the task.

\section{METHOD}

\section{Subjects and Experimental Design}

The Ss were 120 volunteer college students from undergraduate courses. They were assigned in a predetermined random order to the 10 major groupings of the experiment, 12 to each group.

The design was a 5 by 2 factorial, with two 3 by 3 Greco-Latin squares embedded within each of the 10 cells. The factorial design was produced by combining number of memory markers available for S's use (zero, one, two, four, or eight) and conceptual rule defining problem solution (disjunctive or conditional). Each $S$ solved three successive conceptual problems. These three problems correspond to the columns of the Greco-Latin squares. Latin letters were assigned to three conditions of marker valence; when available S's markers were positive only (plus), negative only (minus), or either positive or negative (plus-minus). Greek letters were assigned to the solutions of the three problems. Thus, rows of the Greco-Latin squares correspond to three successive problems, each characterized by a particular solution and a particular set of markers. There were six different rows (two 3 by 3 Greco-Latin squares) and 2 of $12 \mathrm{Ss}$ within each factorial cell were assigned to a row.

\section{Stimulus Materials}

Stimuli used in these problems were geometrical designs drawn on 6-cm-square white cards. The designs varied on four three-valued dimensions: color (red, yellow, and blue); size (small, medium, and large); shape (triangle, square, and circle); and number of identical figures per card (one, two, and three). The 81 possible patterns were arranged in a perceptually unsystematic (Latin square) layout on a $125-\mathrm{cm}-$ square blackboard. Cardboard squares (2-cm square) with a plus sign on one side and a minus sign on the other were used as markers. The $S$ could use either side of a marker only in a plus-minus condition. A pictorial and verbal description of the stimulus population and the general form of solution, disjunctive or conditional, was available to $\mathrm{S}$ throughout the experiment.
Task and Procedure

Instructions included a description of concepts in general, the four dimensions and their values, the rule for solution, the markers (if there were any), and the general procedural requirements of the experiment. A sufficient number of examples of how the rule could be used to combine a pair of attributes to form a concept were given to assure $S$ 's understanding of the general form of solution he was to seek. The $\mathrm{S}$ was told that his task was to find two unknown relevant attributes which, when combined by the given rule, would provide a way of assigning all stimuli on the board to the positive and negative categories of the unknown concept correctly. These were, therefore, attribute identification problems. When markers were available, $S$ was told to use them in any way that he wished to help out in finding the pair of relevant attributes and their use was demonstrated. The $\mathrm{S}$ was not required to use markers, however.

At the beginning of each experimental problem, E pointed to the first card, told $S$ that it was a positive instance and that it contained both unknown relevant attributes. Thereafter, $S$ picked successive instances. After each card was picked, E told S whether it was a positive or a negative instance and asked $S$ for a hypothesis about the solution (the two relevant attributes). Problem solution was operationally defined as three consecutive correct statements of the solution. Any $S$ who had not attained this criterion after 50 card selections in the first problem was eliminated as a nonsolver. Eight Ss, five on conditionals and three on disjunctives, were eliminated on this basis.

\section{RESULTS}

\section{Number of Stimulus Selections to Solution}

Mean numbers of stimulus selections, a measure of overall performance, are presented in Table 1 for the various major conditions of the experiment. An analysis of variance performed on these data revealed the following reliable effects. First, the conditional rule was significantly more difficult than the disjunctive $[F(1,110)=10.38, p<.01]$, despite the fact that Ss were pretrained on the rule to be used. Rule differences decreased after the first problem, however, resulting in a marginal Rule by Ordinal Position of Problems interaction $[F(2,180)=3.52, p<.05]$. Increasing the number of memory markers available to $S$ had a significant effect $[F(4,110)=7.56, p<.01]$, the performance trend showing linear $[F(1,110)=17.34$, $\mathrm{p}<.01]$ and quadratic $[\mathrm{F}(1,110=7.11, \mathrm{p}<.01]$ components. Number of markers available to $S$ interacted with marker variance $[\mathrm{F}(8,180)=4.36$, $p<.01]$, but this effect is primarily attributable to the (logically necessary) lack of difference among valences when no markers were available. Minor effects were attributable to ordinal position of problems $[F(2,180)=3.61, p<.05]$, showing a general improvement in performance with practice on problems of the same type, and to marker valence $[F(2,180)=4.91, p<.01]$, showing an overall tendency for minus and plus-minus markers to be more useful to $S$ than plus only.

Probably the most important source of variance in these data is the Marker Valence by Rule interaction 
$[F(2,180)=8.66, p<.01]$. Especially in the case of conditionals, plus markers lead to inferior performance. Moreover, in disjunctions a mixture of plus and minus markers is better than minus only, while in conditionals minus only are better than a mixture. This is true in general, regardless of the number of markers available or the ordinal position of the problem.

\section{The Use of Memory Markers}

A variety of analyses were performed on measures indexing S's use of memory markers. Groups with no markers were eliminated in these analyses. The measures used were primarily ratios that take account of the differing number of markers available in the various conditions of the experiment. The ratio of number of marker moves to number of possible moves and the ratio of number of markers used to number of markers available are the measures of primary interest. The marker-use measure is the ratio of the maximal number of markers $S$ has on the display board during any problem to the number of markers available to him. For the marker-move ratio, we count the number of times any of the markers was placed on a new stimulus, including its first use on the board.

These measures reveal essentially identical results. With the exception of conditions in which minus markers only were available, none of the major variables of this experiment had any effect on either ratio. In the minus-only conditions, Ss solving conditional problems had a tendency to use more markers and to make more moves than Ss solving disjunctive problems $\left[F_{m o v e}(1,72)=6.53, p<.01\right]$. Ratios increased with the number of minus markers available in both rules, but more for conditionals than disjunctives $\left[F_{\text {move }}(3,72)=\right.$ $3.36, \mathrm{p}<.05$ ] , as shown in Table 2 .

The more markers used and the more moves made with them, the fewer stimulus selections required for solution $\left[\mathrm{r}_{\mathrm{use}}=.43\right.$ and $\mathrm{r}_{\text {move }}=.50$, both ps $\left.<.01\right]$. Ss below the median of either measure require approximately $200 \%$ more trials to solve the problems than those above the median. Means trials for Ss above and below median number of marker moves are 8.1 and 16.8 , respectively. For Ss above and below the median number of markers used the means are 8.3 and 16.6, respectively. Differences are statistically significant $\left[F_{\text {move }}(1,104)=43.19, p<.01\right]$. Furthermore, there were interactions between rule and median split and between number of markers available and median split. A greater difference obtained between above and below Ss in overall performance on the conditional than on the disjunctive rule $[F(1,104)=8.81, p<.01]$. Moreover, the difference between above and below median Ss increased with number of markers available $[F(3,104)=4.76, p<.01]$. While there were similar trends in plus-only and plus-minus marker conditions, analogous effects were not statistically significant.

Further analysis was undertaken on the use of plus (or
Table 2

Measures of Marker Use and Moves When Negative Markers Are Available

\begin{tabular}{llllll} 
& & \multicolumn{4}{c}{ Number } \\
\cline { 3 - 6 } & Measure & 1 & 2 & 4 & 8 \\
\hline \multirow{2}{*}{ Disjunctive Rule } & Move & .25 & .25 & .28 & .29 \\
& Use & .63 & .65 & .71 & .73 \\
Conditional Rule & Move & .30 & .33 & .34 & .37 \\
& Use & .64 & .70 & .75 & .81 \\
\hline
\end{tabular}

minus) sides of the markers available in the plus-minus condition. In an analysis of the percentage (per S) of plus side use to total marker use, the rule effect was reliable $[F(1,104)=10.21, p<.01]$. While there was little difference between the number of plus and minus markers used on the disjunctive problems ( $48 \%$ and $52 \%$, respectively), minus markers were significantly more frequent on conditional problems (27\% plus and $73 \%$ minus). When Ss are divided at the median of overall performance within each rule condition, Ss above the median used $25 \%$ positives, while Ss below the median used $19 \%$ positives in conditional problems. Corresponding percentages in disjunctive problems were $51 \%$ and $45 \%$. Mean numbers of card selections to solution for Ss above and below the median in using the plus side in the plus-minus conditions are 4.1 and 3.7 , respectively, for disjunctives and 11.7 and 6.1, respectively, for conditionals.

Several trends were observed in an examination of types of marker moves. When both plus and minus markers were available, Ss tended to shift more frequently from positives to negatives, but the difference was reliable only for conditionals (plus to minus, $32 \%$; minus to plus, $15 \%$ of total moves) $[\mathrm{F}(1,335)=7.81$, $p<.01]$. This analysis was limited to the first eight stimulus selections, after which the predominant use of minus markers tended to obscure any effects. Ss who scored below the median number of card selections to solution not only made relatively more shifts in markers but also made more shifts to negative instances (plus to minus and minus to minus shifts) than Ss above the median $(77 \%$ and $47 \%$, respectively) $[\mathrm{F}(1,30)=10.91$, $p<.01]$. No reliable difference was observed for disjunctive problems.

\section{Selection Strategies}

Another way to study individual differences among Ss is an analysis of selection strategies (Laughlin, 1968). The expectation is that, for these problems, a more systematic pattern of selection should characterize the better $\mathbf{S}$. In this task a conservative focusing strategy amounts to using the first instance (positive) as a basis for subsequent stimulus selections, which are chosen to differ only in a single attribute. This is a simple and efficient strategy for solving conjunctive attribute identification problems. In fact, given the limits on 
human memory and inference, a sequence of instances that minimizes the amount of stimulus change would seem intuitively and in a psychological sense to optimize information utilization, regardless of the conceptual rule. Thus, the analysis adopted here was basically an assessment of interstimulus variability in S's selection pattern. It entailed simply counting the number of dimensions that changed values between one selection and another and averaging overall selections. While there is a variety of options available (Bourne, in press), we chose four to work with: (1) All selections by S were compared to the first instance, a positive instance in all cases; (2) selections were compared to the most recent positive instance; (3) and (4) were analogous comparisons to the first and the just-preceding negative instance in the series. In analyses of variance, 2 and 4 were the only two measures to show any discriminating effects. Higher variability scores were obtained by Ss who were above the median number of card selections to solution. The difference between above (2.53) and below (2.44) median Ss was reliable for Measure 2 only in disjunctive concepts $[\mathrm{F}(1,80)=4.01, \mathrm{p}<.05]$. In contrast, the difference (above, 2.78; below, 2.63) was significant only for conditional problems when Measure 4 was analyzed $[F(1,80)=6.86, p<.05]$. One implication is that the better Ss tend to adopt a shifting focus strategy. The focus shows a slight tendency to be on positive instances in disjunctive problems and a distinct tendency to be on negative instances in conditionals. This pattern is particularly evident when S tries to retain in his own memory (infrequent use of markers) all information provided by stimulus selections. For Ss below the median in their use of markers, the mean 2 focusing scores on disjunctions were 2.37 and 2.53 for the better and poorer Ss, respectively. For those above the median in their use of markers, there is practically no difference in focusing scores (2.51 and 2.53) between the better and the poorer Ss. The corresponding Measure 4 focusing scores on conditionals were 2.73 and 2.77 for Ss who used the markers and 2.54 and 2.78 for those who fell below the median in marker usages. Thus, as $\mathrm{S}$ retains more information through his use of markers, stimulus variability or focusing becomes less important and the variability in card selections increases.

\section{DISCUSSION}

The availability of previously seen or selected stimuli plus their category assignments facilitates performance in attribute identification problems (e.g., Bourne et al, 1969). This effect is confirmed in the present study under conditions in which $S$ is allowed to adopt, up to controlled limits, his own strategy for the use of memory markers. The more markers available, the better is performance. This is true in general, regardless of the rules or the type of markers available. Obviously, however, markers facilitate the performance of only those Ss who use them.
Some strategies for using markers are better than others. The analysis of Bruner et al (1956) and Laughlin (1968) might be taken to suggest the superiority of a fixed over a shifting focus strategy. In the present study, Ss who moved the markers around on the board to successively selected and more informative instances did better. Thus, in the present study, the better Ss appear to have used a shifting focus strategy, comparing their successive selections to those just previously examined. Alternatively, it might be argued that marker usage is mainly an index of the degree to which S "actively participates" in the task. Rather than a difference in cognitive strategy then, the critical effect is one of degree of $S$ involvement. The fact that moves made by "active" Ss tended to be from lesser to more informative instances casts some doubt on a simple level of activity interpretation, however.

The kind of markers available (plus, minus, or a mixture of plus and minus markers) made relatively little difference in disjunctive problems. In the condition where both kinds of markers were available, Ss were indifferent, tending to choose roughly equal numbers of plus and minus sides. Choice was not affected by whether $S$ was above or below median in the overall performance on the task. This might be viewed as inconsistent with results of Bourne and Guy (1968), who observed better performance on disjunctive problems with positive instances than with negatives or a mixture. Differences among instances were not large in either study, however. If the discrepancy in results is significant, it is probably due to the fact that a study-test reception paradigm, in which stimuli available to $\mathrm{S}$ are predetermined, was used earlier, while a selection paradigm, in which $S$ is completely free to choose and mark stimuli, was used in the present study. The selection paradigm permits greater freedom in the use of stimulus information that is probably especially facilitative in conditions that provide a mixture of instances.

In contrast to disjunctives, minus markers were much more commonly used when the choice was available in conditional problems. Performance in the minus-only condition was significantly superior to performance in the plus or plus-minus conditions. Furthermore, Ss who fell above the median in overall performance tended to use more minus markers in conditional problems.

These results are consistent with the observations of Bourne et al (1969) and Bourne and Guy (1968) showing the availability of negative instances to be significantly more facilitative than positive instances in conditional problems. Their interpretation was that negative instances are a smaller, more homogeneous category than positive instances in a conditional problem and, therefore, easier to find communalities among and to make inferences from. The results of the present experiment are consistent with that interpretation. Furthermore, over three successive problems, Ss learn to recognize the importance of negative instances for identifying the relevant attributes of conditional 
problems, leading them to mark negative instances with increasing frequency in preference to positive.

Despite the fact that the rule was known to $S$ at the outset, conditional problems were significantly more difficult than disjunctive. This is not a new finding, and a number of interpretations have been offered, such as those based on the structural complexity of concepts (Neisser \& Weene, 1962) and on the cognitive requirements of appropriate strategies (Laughlin, 1968). The available data failed to distinguish among these possibilities. It is worth noting, however, that the difference between rules is reduced both by increasing the number of markers available and with successive problems. The latter result is consistent with the idea that factors contributing to differences in rule difficulty are largely cognitive and can be overcome by practice, although Taplin (1971) reported a significant difference between conditionals and disjunctives even after 25 problems.

Finally, marker usage tends to be directly associated with variability in S's stimulus selections. Simple, conservative, low-variability selection patterns reduce the memory requirements of a task (Bruner et al, 1956). In the extreme case, where each pattern differs in only one dimension from a focus or from the preceding pattern, $S$ needs only to maintain a composite hypothesis, eliminating each dimension as it is proved irrelevant. When $S$ has some artificial means of remembering previous patterns and of making comparisons between new selections and those that were previously chosen, the need for a conservative approach is reduced. The availability and use of markers provide an artificial memory, or bookkeeping system, that is the likely basis for the relationship between these variables and variability in stimulus selection.

\section{REFERENCES}

Bourne, L. E., Jr. Effects of rule, memory, and truth-table information on attribute identification. Journal of Experimental Psychology, in press.

Bourne, L. E., Jr., \& Guy, D. E. Learning conceptual rules: II. The role of positive and negative instances. Journal of Experimental Psychology, 1968, 77, 488-494.

Bourne, L. E., Jr., Ekstrand, B. R., \& Montgomery, B. Concept learning as a function of the conceptual rule and the availability of positive and negative instances. Journal of Experimental Psychology, 1969, 82, 538-544.

Bourne, L. E., Jr., Goldstein, S., \& Link, W. E. Concept learning as a function of availability of previously presented information. Journal of Experimental Psychology, 1964, 67, 439-448.

Bruner, J. S., Goodnow, J. J., \& Austin, G. A. A study of thinking. New York: Wiley, 1956.

Hovland, C. I. A "communication analysis" of concept learning. Psychological Review, 1952, 59, 461-472.

Laughlin, P. R. Focusing strategy for eight conceptual rules. Journal of Experimental Psychology, 1968, 77, 661-669.

Levine, M. Hypothesis behavior by humans during discrimination learning. Journal of Experimental Psychology, 1966, 71, 331-338.

Neisser, U., \& Weene, P. Hierarchies in concept attainment. Journal of Experimental Psychology, 1962, 66, 640-645.

Pishkin, V., \& Wolfgang, A. Number and type of available instances in concept learning. Journal of Experimental Psychology, 1965, 69, 5-8.

Taplin, J. E. An experimental study of human reasoning and conceptual behavior. Unpublished $\mathrm{PhD}$ dissertation, University of Adelaide, Australia, 1971.

(Received for publication August 10, 1973; revision received August 27, 1973; revision accepted September 22, 1973.) 